\title{
Intact spatial updating with severely degraded vision
}

\author{
Margaret R. Tarampi, Sarah H. Creem-Regehr, \\ AND WILliam B. THOMPSON \\ University of Utah, Salt Lake City, Utah
}

\begin{abstract}
Critical to low-vision navigation are the abilities to recover scale and update a 3-D representation of space. In order to investigate whether these abilities are present under low-vision conditions, we employed the triangulation task of eyes-closed indirect walking to previously viewed targets on the ground. This task requires that the observer continually update the location of the target without any further visual feedback of his/her movement or the target's location. Normally sighted participants were tested monocularly in a degraded vision condition and a normal vision condition on both indirect and direct walking to previously viewed targets. Surprisingly, we found no difference in walked distances between the degraded and normal vision conditions. Our results provide evidence for intact spatial updating even under severely degraded vision conditions, indicating that participants can recover scale and update a 3-D representation of space under simulated low vision.
\end{abstract}

Low-vision individuals navigate the world under visually degraded conditions in which the visual information that they receive is impaired by the nature of their visionbased disability. Although adaptive and low-vision devices can be used to help these individuals perform daily tasks such as reading, writing, and cooking, many lowvision individuals rely on their remaining vision and their other senses for spatial orientation and navigation, rather than relying on external aids (Ludt \& Goodrich, 2002). Existing research on low-vision navigation has primarily focused on obstacle avoidance while walking (Kuyk, Elliott, Biehl, \& Fuhr, 1996; Long, Rieser, \& Hill, 1990; Marron \& Bailey, 1982; Pelli, 1987). This work does not directly address the ability to recover absolute distance and the overall scale of the space under low-vision conditions, since the constant feedback from eyes-open walking allows obstacle-avoidance strategies involving only relative distance and time-to-collision. The ability to recover scale is critical for components of navigation, such as path planning and spatial updating of locations with movement. For this reason, our own work focuses on the perception of absolute distances under severely degraded vision. Surprisingly, our preliminary work demonstrated that observers can accurately indicate distances ranging from 2 to $3.5 \mathrm{~m}$ in a degraded visual setting in which the target is just barely detectable (Tarampi, Creem-Regehr, \& Thompson, 2008). Our present goal was to provide more definitive evidence that observers in a simulated low-vision condition could recover a perceptual representation that would support the complex goal of keeping track of their location in the environment.
Although vision provides a rich set of sources of information for relative distance, only a few of these visual cues directly indicate absolute size and distance. Beyond distances of a few meters from the viewer, the primary visual information for scale comes from familiar size and body-scaled perspective information, although observerinduced motion parallax may also play a role. Low vision, particularly when it involves severely degraded acuity and contrast, almost certainly impacts familiar size, although there has been little if any controlled study of this. Reductions in acuity and contrast affect perspective-based visual information by reducing high-frequency detail used to infer vanishing points and lines and texture gradients. Depending on the nature of the environment under view and the degree of degradation, this would be predicted to negatively affect both the accuracy and precision of distance judgments. The potential impact of low vision on motion parallax is less clear, although there is some evidence that it provides at best a weak indication of absolute distance (Beall, Loomis, Philbeck, \& Fikes, 1995).

Assessing the perceptual capabilities of low-vision individuals has theoretical implications for both the nature of perception and action and for rehabilitation and the design of environmental spaces. Many studies have utilized visually directed walking measures as indications of perceived distance, showing that observers are quite accurate at walking without vision to previously viewed targets under full-cue conditions (Loomis, Da Silva, Fujita, \& Fukusima, 1992; Philbeck \& Loomis, 1997; Rieser, Ashmead, Talor, \& Youngquist, 1990; Thomson, 1983). Direct walking involves walking without vision along a straight path to a target. In principle, the direct walk-

M. R. Tarampi, margaret.tarampi@psych.utah.edu 

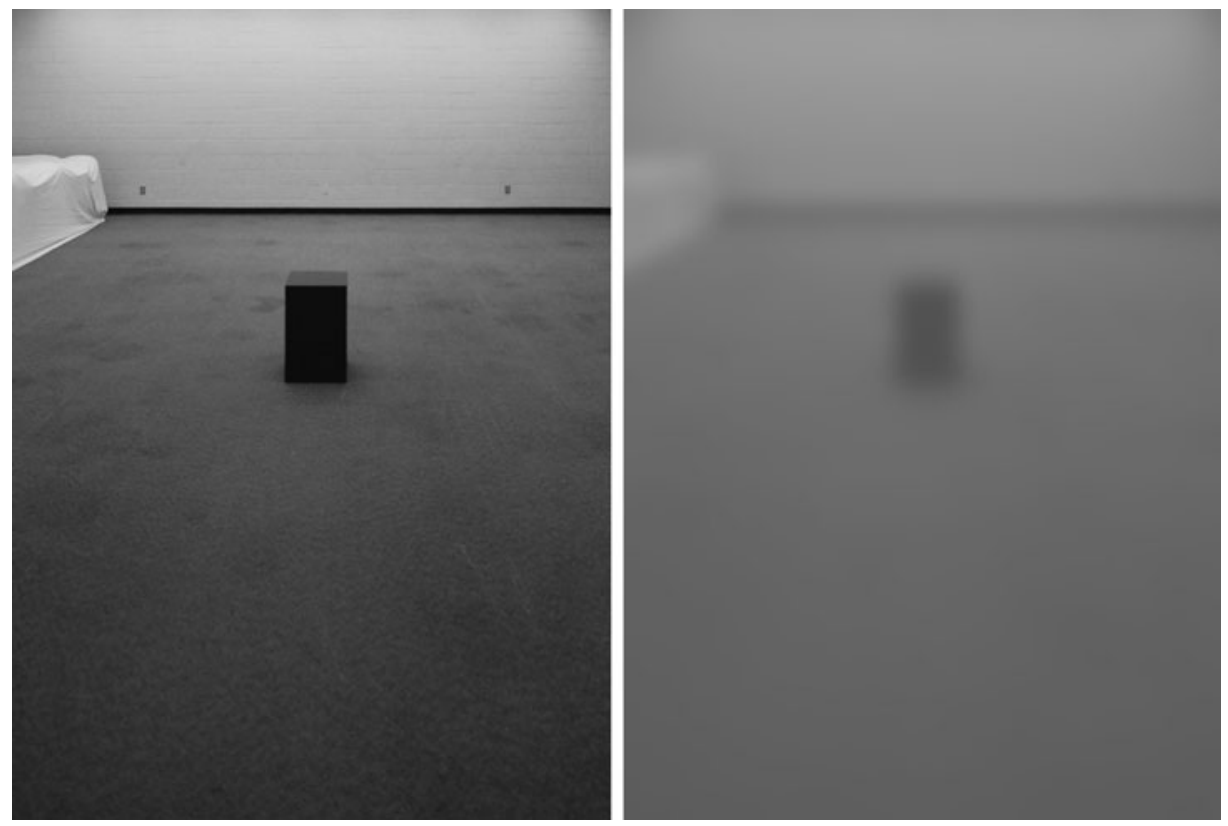

Figure 1. Simulation of the participant's view in normal (left) and degraded (right) conditions.

ing task can be accomplished with motor preplanning or other processes that do not require continuous updating of the perceptual representation of the space being traveled through. A triangulation task, such as indirect walking, involves walking without vision in a direction other than toward the target and, when cued, turning and walking to the target location. This more complex and unpredictable task would make it difficult for the observer to use preplanning strategies, and serves as a good test of the continuous updating of self-location with respect to the environment. Our present comparison of direct and indirect walking measures under degraded and normal vision conditions (see Figure 1) provided a means to test whether a perceptual representation could be recovered that supported more complex navigation than was originally demonstrated in Tarampi et al. (2008).

\section{METHOD}

\section{Participants}

A total of 20 psychology students from the University of Utah participated as part of a course requirement or for extra credit. Ten (6 males and 4 females, mean age 21.8 years) participated in Experiment 1 comparing degraded and normal viewing conditions. Ten others ( 6 males and 4 females, mean age 21.5 years) participated in Experiment 2, a normal viewing condition which served as a control for possible order effects, as described below. All participants had normal or corrected-to-normal vision.

\section{Materials}

Each participant wore noise-canceling headphones connected to a receiver unit and an MP3 player that played pink noise to mask environmental noise. The experimenter administered instructions through a wireless microphone. For the degraded vision condition, the participant wore welding goggles with a theatrical lighting gel (ROSCO Cinegel \#3047: Light Velvet Frost), resulting in an averaged tested acuity of $\log$ MAR $1.53(S D=0.16)$, and an averaged tested contrast sensitivity of $\log 0.36(S D=0.28)$ (see Figure 2). For the normal vision condition, the participant wore welding goggles with clear flat plastic lenses. Both sets of goggles allowed for monocular viewing only, through the right eye aperture, and had a tested field of view from the circular aperture of $72^{\circ}$ in the horizontal and $68^{\circ}$ in the vertical. The left eye was blacked out. In both conditions, the participant wore a hood made of blackout cloth that was lowered before the participant walked to block the view of the target, environment, and surrounding light cues. The targets were two sizes of black matte boxes $(51 \mathrm{~cm} \mathrm{H} \times 32 \mathrm{~cm}$ $\mathrm{W} \times 32 \mathrm{~cm} \mathrm{D} ; 56 \mathrm{~cm} \mathrm{H} \times 36 \mathrm{~cm} \mathrm{~W} \times 36 \mathrm{~cm} \mathrm{D})$. The experiment room had $7.7 \mathrm{~m} \times 10.5 \mathrm{~m}$ of walkable space and was approximately evenly lit.

\section{Design}

Experiment 1 used a 2 (condition) $\times 2$ (task) $\times 3$ (distance) factorial design in which viewing condition (degraded or normal), task (direct or indirect walking), and distance were within-subjects variables. Experiment 2 used a 2 (task) $\times 3$ (distance) $\times 2$ (order)

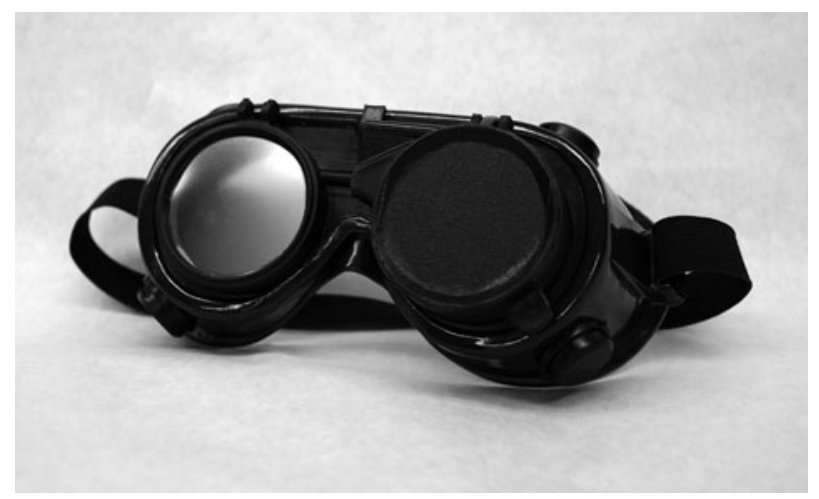

Figure 2. Degraded-vision goggles: welding goggles with the left eye occluded and a theatrical lighting gel in the right eye to impair vision. 
design, with order as a between-subjects variable and task and distance as within-subjects variables.

\section{Procedure}

Each participant was first trained in a space separate from the experiment room. The training included (1) walking without vision while being guided by the experimenter, (2) blind walking independently, (3) practice with the direct and indirect walking tasks, and (4) practice raising/lowering the hood. None of the training steps involved feedback about the accuracy of walking to target locations. After completing the training, the participant put on the degraded-vision goggles and a hood over them and was led into the experiment room without vision. To ensure that cues related to familiarity of the space were not utilized, participants remained naive to the experiment room until after the degradedvision condition.

The experimenter guided each participant to the starting location. Participants then performed 2 practice trials without feedback to ensure that they understood the experimental instructions. Following the 2 practice trials, the participants performed 14 additional trials. In each trial, a target was located on the ground plane at egocentric distances of $1.5,3.1$, or $6 \mathrm{~m}$, for a total of 2 trials at each distance for each task (direct or indirect walking). One dummy trial was also included at each of the distances of 4 and $5 \mathrm{~m}$ in order to make the target trial distances less predictable. The task, target size, and target distances were randomized across trials. The participant was allowed to view the target and its location in the environment as well as an LED presented at about eye level on the right-side wall for a total of $5 \mathrm{sec}$. The experimenter then instructed the participants to lower their hoods. Once this was done, the participants were told which task to perform with the verbal cue "direct" or "indirect." For all trials, the participants were told to walk to the target, which would be moved by an experimenter so that they would not collide with it. At the end of each trial, the participants were guided blindfolded along a circuitous path back to the starting location as the next target was placed for the following trial. Participants did not receive any performance feedback.

On a direct walking trial, the participants walked without vision until they reached the target location. The participants' final positions were marked with stickers on the ground between participants' feet. The $x$ - and $y$-coordinates of this endpoint were recorded using metric measuring tapes along the $x$ - and $y$-axes. On an indirect walking trial, the participants first walked in an oblique direction without vision toward the previously seen LED. When prompted, they paused and turned toward the perceived target, then walked the rest of the way to the target without vision. The turning point and the endpoint were marked with stickers on the ground. The $x$ - and $y$-coordinates of both the turning location and the endpoint were recorded.

Upon completion of the degraded-vision condition, the participants were led out to the hallway blindfolded and given a 5-min break without the goggles to counteract any adaptation that may have occurred. Then, they put on the normal-vision goggles and the hoods. Blindfolded, the participants were then led into the experiment room to complete the normal trials. Upon finishing the trials in the normal condition, each participant performed two vision tests. The participant's logMAR acuity (ETDRS Acuity Chart) and logcontrast sensitivity (Pelli-Robson Contrast Sensitivity) were first tested monocularly with the degraded-vision goggles used in the experiment and then with the normal-vision goggles.

In Experiment 1, the normal condition was always performed after the degraded condition to prevent the participants from having full-cue experience with the room before making their low-vision judgments. Consequently, judgments in the normal condition may have been influenced by the prior block of degraded-vision trials; we therefore conducted a second experiment involving only the normal condition, to compare judgments made in the first block of trials with those made in the second. Other than having only one block of normal viewing trials, all other protocols in Experiment 2 were identical to the protocol of Experiment 1. The normal condition in Experiment 2 allowed for a between-subjects comparison with the normal condition of Experiment 1 to test for order effects.

\section{RESULTS}

Overall walking accuracy was essentially the same in the degraded and normal viewing conditions and did not vary as a function of the direct versus indirect walking task (see Figures 3 and 4). Although distance estimates were slightly underestimated in both conditions, Experiment 2 showed that this was not a result of factors associated with performing two blocks of trials, since there was no difference in performance as a function of order. For each participant, target distance, and task, we calculated the average turn point (when applicable) and endpoint by averaging the $x$ - and $y$-coordinates separately. The originto-endpoint distance (OE) was calculated in order to compare performance between the direct and indirect walking tasks. In Experiment 1, a 2 (condition) $\times 2$ (task) $\times$ 3 (distance) ANOVA was performed on the mean OE with condition, task, and distance as within-subjects variables. There was a significant effect only of distance $[F(2,18)=$ $285.230, p<.0001, \eta_{\mathrm{p}}^{2}=0.969$ ], showing that distance walked increased with increasing absolute distance. Notably, there was no difference in OE between the degraded and normal viewing conditions ( $p=.216)$ and no interaction of viewing condition and task ( $p=.267)$. In Experiment 2 , a 2 (order) $\times 2$ (task) $\times 3$ (distance) ANOVA was performed on the mean OE with order (normal condition presented first or second) as a between-subjects variable and task and distance as within-subjects variables. The only significant effect was of distance $[F(2,36)=$

\section{Average Across Participants: Direct Walking}

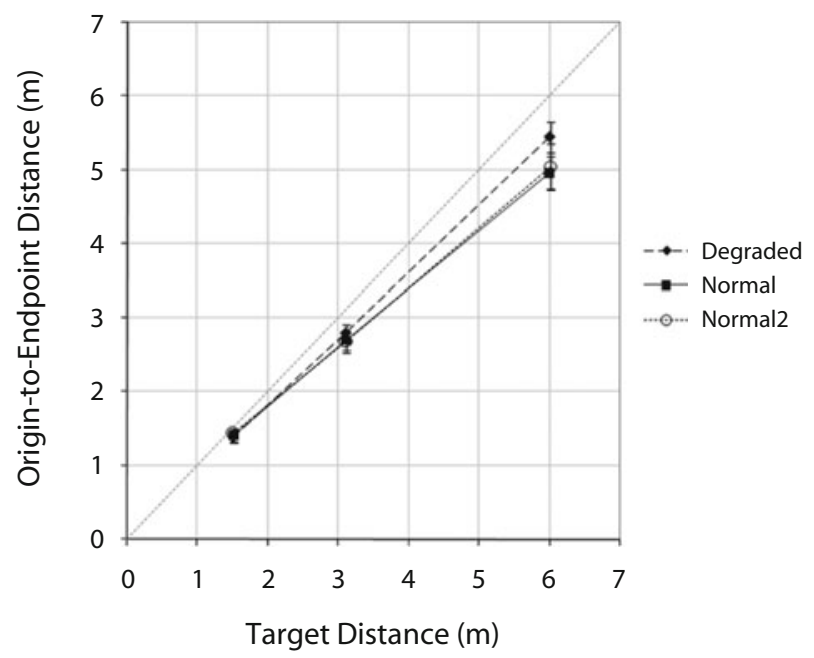

Figure 3. Mean distances from the origin to the endpoint, averaged across observers for the direct walking task in the degraded and normal conditions (Experiment 1) and the normal condition (Experiment 2). Error bars represent \pm 1 standard error of the mean. 


\section{Average Across Participants: Indirect Walking}

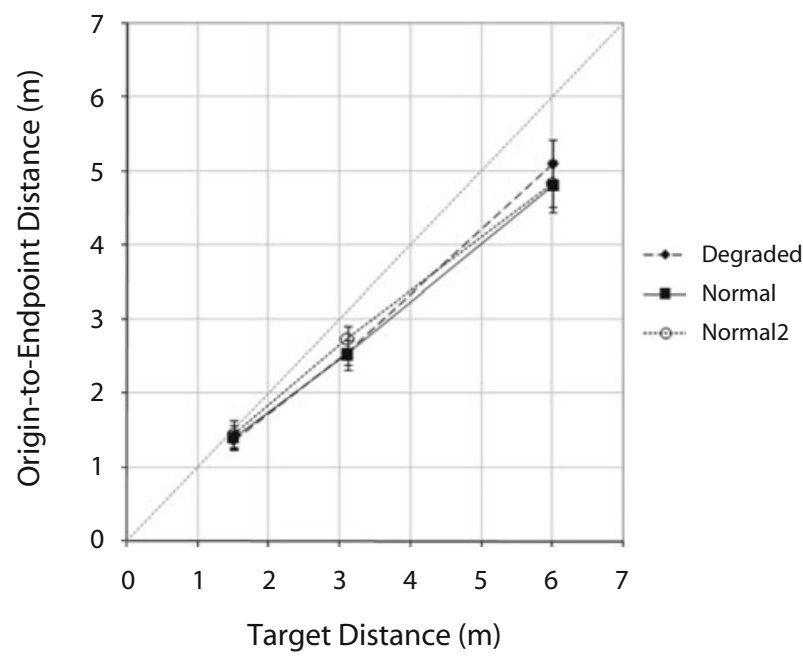

Figure 4. Mean distances from the origin to the endpoint, averaged across observers for the indirect walking task in the degraded and normal conditions (Experiment 1) and the normal condition (Experiment 2). Error bars represent \pm 1 standard error of the mean.

$\left.367.988, p<.0001, \eta_{\mathrm{p}}^{2}=0.953\right]$. There was no effect of order $(p=.770)$.

Although constant error was not affected by the viewing condition manipulation, we did find a difference in precision. Variable error, as a measure of precision, was calculated as the $S D$ of the mean distances walked at each distance for each participant in each task and condition. The same ANOVA as described above was performed on variable error for Experiment 1, showing a significant effect of viewing condition $\left[F(1,9)=6.744, p<.05, \eta_{\mathrm{p}}^{2}=\right.$ $0.428]$, with greater variability in the degraded $(M=$ $.302)$ than in the normal $(M=.212)$ condition. Consistent with other blind walking studies, there was also significant increase in variable error with increasing distance $\left[F(2,18)=7.037, p<.01, \eta_{\mathrm{p}}^{2}=0.439\right]$.

To test whether the $x$ - and $y$-coordinates may have been affected differently by the visual conditions and tasks, we conducted a repeated measures MANOVA analyzing the two coordinates as a linear combination. Condition, task, and distance were within-subjects variables for Experiment 1 . The MANOVA revealed a main effect of task $\left[F(2,8)=5.252, p<.05, \eta_{\mathrm{p}}^{2}=0.568\right]$ and distance $\left[F(4,36)=84.616, p<.0001, \eta_{\mathrm{p}}^{2}=0.983\right]$ and no significant interactions with condition. The effect of task is further qualified by the univariate analysis, which revealed a significant effect of the $x$-coordinate for task $[F(1,9)=$ $\left.11.247, p<.008, \eta_{\mathrm{p}}^{2}=0.555\right]$ but not of the $y$-coordinate. This reflects the tendency for participants to overshoot the $x$-coordinate in the indirect walking task $(M=-3.08805$ and -0.8674 , for indirect and direct, respectively). There was also a main effect of the $y$-coordinate for distance $\left[F(2,18)=270.193, p<.0001, \eta_{\mathrm{p}}^{2}=0.968\right]$, consistent with the finding that the location of the $y$-coordinate increased with target distance.

\section{DISCUSSION}

We provide evidence that absolute distance estimation under conditions of severely reduced acuity and contrast remains accurate, though with increased variability compared with nondegraded viewing. The utilization of the indirect walking task provides additional evidence that the perceptual representation of distance was sufficient to allow for the continuous updating of the environment during walking. Since our experimental procedure involved blob-like target objects with no obvious familiar size information and limited head translation during viewing, the most plausible explanation for the good performance under our low-vision conditions is that the participants were still able to use body-scaled perspective information. Given the impoverished nature of the visual environment, we believe that this was most likely eye-height scaled angle of declination information based on the angle from the observer's eye height to a target located on the ground plane (Ooi, Wu, \& He, 2001; Sedgwick, 1986); it is still unclear whether or not visual information was used as the reference for the horizontal. As seen in Figure 1, even under the low-vision conditions there was visual information for the boundary between the floor and wall. It will be important to determine whether information such as this is critical to making accurate distance judgments. Questions of this sort have broader implications for making architectural spaces visually accessible.

In addition to the main finding of comparable accuracy in spatial updating across the viewing conditions, there were several interesting additional outcomes of the distance estimation tasks. In Experiment 1, performance on the direct and indirect walking tasks in the normal condition was less accurate than expected, showing underestimation at further distances. We replicated these findings in Experiment 2 and ruled out order effects. It is possible that the underestimation is due to the unpredictability of the task during the viewing period, since observers did not know whether they would perform the indirect or direct walking task until after their eyes were closed. There may be differences in the representation of visual information when the task is unknown, or there may also be cognitive interference from the other task. Previous studies with multiple-task methodologies have shown underestimation of distance consistent with our present results (Loomis, Klatzky, Philbeck, \& Golledge, 1998; Philbeck, Loomis, $\&$ Beall, 1997), but a clear understanding of the causes will require further examination.

A second effect seen in both viewing conditions was the significant overshoot to the left in the $x$-coordinate for the indirect walking task. One speculative explanation for this bias could be right-eye monocular viewing. We chose to test monocularly with the right eye instead of monocularly with the observer's dominant eye because the degradedvision filter could not be precisely replicated in both eyes. It is also possible that there is a direction bias in the indirect walking task that is dependent on the heading direction of the first leg. This bias is evident but not discussed in previous studies (He, Wu, Ooi, Yarbrough, \& Wu, 2004; Philbeck 
et al., 1997). Observers in our experiment always walked initially in a direction to their right, a procedure chosen because of the configuration of the room. Future investigation could explore the influence of monocular viewing and initial heading direction on directional walking biases.

Despite our degrading vision to a range between profound low vision and near-blindness, observers still performed the visually guided action-based measures of direct and indirect walking to previously viewed targets as accurately as in the normal condition. Our results provide evidence that participants can recover scale and update a perceptual representation of space under simulated low vision, at least to distances of several meters out. Our finding of increased variability in responses may be attributed to increased uncertainty in one's self-position during updating, given the reduced visual context available during initial viewing of the environment. Precision in updating could be facilitated with the presence of multiple targets, as shown in a full-cue study that found that the context of nearby landmarks reduced response variability in blind walking (Philbeck \& O'Leary, 2005). Also, although observers under low-vision conditions can accurately update their self-positions with respect to one object, they may not be able to update multiple locations at the same time, or they may use different memory strategies under fullcue versus degraded-vision conditions. Further investigation is necessary to generalize these results to the updating of multiple target locations and to examine how precision might be improved.

\section{AUTHOR NOTE}

This work was supported by NIH Grant 1 R01 EY017835-01. Correspondence concerning this article should be addressed to M. R. Tarampi, Department of Psychology, University of Utah, 380 S. 1530 E., Room 502, Salt Lake City, UT 84112 (e-mail: margaret.tarampi@psych .utah.edu).

\section{REFERENCES}

Beall, A. C., Loomis, J. M., Philbeck, J. W., \& Fikes, T. J. (1995, February). Absolute motion parallax weakly determines visual scale in real and virtual environments. Paper presented at the Conference on Human Vision, Visual Processing, and Digital Display, San Jose, CA.

He, Z. J., Wu, B., OoI, T. L., Yarbrough, G., \& Wu, J. (2004). Judging egocentric distance on the ground: Occlusion and surface integration. Perception, 33, 789-806. doi:10.1068/p5256a
KuyK, T., Elliott, J. L., Biehl, J., \& Fuhr, P. S. (1996). Environmental variables and mobility performance in adults with low vision. Journal of the American Optometric Association, 67, 403-409.

LONG, R. G., RIESER, J. J., \& HiLL, E. W. (1990). Mobility in individuals with moderate visual impairments. Journal of Visual Impairment \& Blindness, 84, 111-118.

Loomis, J. M., Da Silva, J. A., Fujita, N., \& Fukusima, S. S. (1992). Visual space perception and visually directed action. Journal of Experimental Psychology: Human Perception \& Performance, 18, 906921. doi:10.1037/0096-1523.18.4.906

Loomis, J. M., Klatzky, R. L., Philbeck, J. W., \& Golledge, R. G. (1998). Assessing auditory distance perception using perceptually directed action. Perception \& Psychophysics, 60, 966-980.

LudT, R., \& Goodrich, G. L. (2002). Change in visual perception detection distances for low vision travelers as a result of dynamic visual assessment and training. Journal of Visual Impairment \& Blindness, 96, 7-21.

Marron, J. A., \& Bailey, I. L. (1982). Visual factors and orientationmobility performance. American Journal of Optometry \& Physiological Optics, 59, 413-426.

OoI, T. L., Wu, B., \& HE, Z. J. (2001). Distance determined by the angular declination below the horizon. Nature, 414, 197-200. doi:10 $.1038 / 35102562$

Pelli, D. G. (1987). The visual requirements of mobility. In G. C. Woo (Ed.), Low vision: Principles and applications (pp. 134-146). Berlin: Springer.

Philbeck, J. W., \& Loomis, J. M. (1997). Comparison of two indicators of perceived egocentric distance under full-cue and reduced-cue conditions. Journal of Experimental Psychology: Human Perception \& Performance, 23, 72-85. doi:10.1037/0096-1523.23.1.72

Philbeck, J. W., Loomis, J. M., \& Beall, A. C. (1997). Visually perceived location is an invariant in the control of action. Perception \& Psychophysics, 59, 601-612.

Philbeck, J. W., \& O'Leary, S. (2005). Remembered landmarks enhance the precision of path integration. Psicologica, 26, 7-24.

Rieser, J. J., Ashmead, D. H., Talor, C. R., \& Youngquist, G. A. (1990). Visual perception and the guidance of locomotion without vision to previously seen targets. Perception, 19, 675-689. doi:10.1068/ p190675

SEDGWICK, H. A. (1986). Space perception. In K. R. Boff, L. Kaufman, \& J. P. Thomas (Eds.), Handbook of perception and human performance (Vol. 1, pp. 1-57). New York: Wiley.

Tarampi, M. R., Creem-Regehr, S. H., \& Thompson, W. B. (2008). Visually directed walking to targets viewed with severely degraded vision is surprisingly accurate [Abstract]. Journal of Vision, 8, 754.

THOMson, J. A. (1983). Is continuous visual monitoring necessary in visually guided locomotion? Journal of Experimental Psychology: Human Perception \& Performance, 9, 427-443. doi:10.1037/0096 $-1523.9 .3 .427$

(Manuscript received July 14, 2009; revision accepted for publication August 28, 2009.) 